Mappemonde

Revue trimestrielle sur l'image géographique et les

formes du territoire

$122 \mid 2017$

Varia

\title{
Le Blue Lagoon (Islande), une hétérotopie érigée en marque
}

Lionel Laslaz

(2) OpenEdition

Journals

Édition électronique

URL : http://journals.openedition.org/mappemonde/563

DOI : $10.4000 /$ mappemonde.563

ISSN : $1769-7298$

Éditeur

UMR ESPACE

Référence électronique

Lionel Laslaz, «Le Blue Lagoon (Islande), une hétérotopie érigée en marque », Mappemonde [En ligne], 122 | 2017, mis en ligne le 08 décembre 2017, consulté le 15 septembre 2020. URL : http:// journals.openedition.org/mappemonde/563

Ce document a été généré automatiquement le 15 septembre 2020.

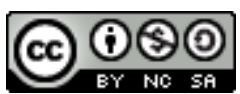

La revue Mappemonde est mise à disposition selon les termes de la Licence Creative Commons Attribution - Pas d'Utilisation Commerciale - Partage dans les Mêmes Conditions 4.0 International. 


\title{
Le Blue Lagoon (Islande), une hétérotopie érigée en marque
}

\author{
Lionel Laslaz
}

1 En Islande, le Blue Lagoon illustre la volonté des acteurs économiques (plus largement que touristiques) de proposer un produit en décalage avec l'image habituelle de l'île (par la couleur, la chaleur), tout en étant ancré dans les pratiques des habitants (bains chauds) et les attendus des touristes (paysages chaotiques de lave). Ce site hétérotopique est devenu une véritable marque (déposée) et un produit d'appel du tourisme. Toutefois, son coût prohibitif et son aspect artificiel font que sa part relative dans les sites islandais fréquentés recule.

\section{L'essor rapide post-crise du tourisme en Islande}

\section{La crise comme outil de l'envol touristique}

2 Avec 1,7 million de touristes en 2016 (trois fois plus qu'en 2010), l'Islande connait un développement d'activité sans précédent et sans guère d'équivalent. Celle-ci représente désormais $20 \%$ du PIB de l'île (les dépenses des visiteurs étrangers augmentent de $31 \%$ par an), quand la puissance publique, avant la crise, manifestait une certaine perplexité pour le développement du tourisme (Jóhannesson et Huijbens, 2010). Depuis 2000, l'île compte plus de visiteurs que d'habitants (338 349 au $1^{\text {er }}$ janvier 2017, soit une hausse de 1,8\% par rapport à l'année précédente) (Laslaz, 2016). Depuis 2010, le nombre de visiteurs a crû en moyenne de $21,6 \%$ par an et les nuitées ont doublé en sept ans (6,5 millions en 2015 contre 3 millions en 2009) (figure 1). 
Figure 1. Nombre de visiteurs étrangers en Islande entre 1949 et 2016

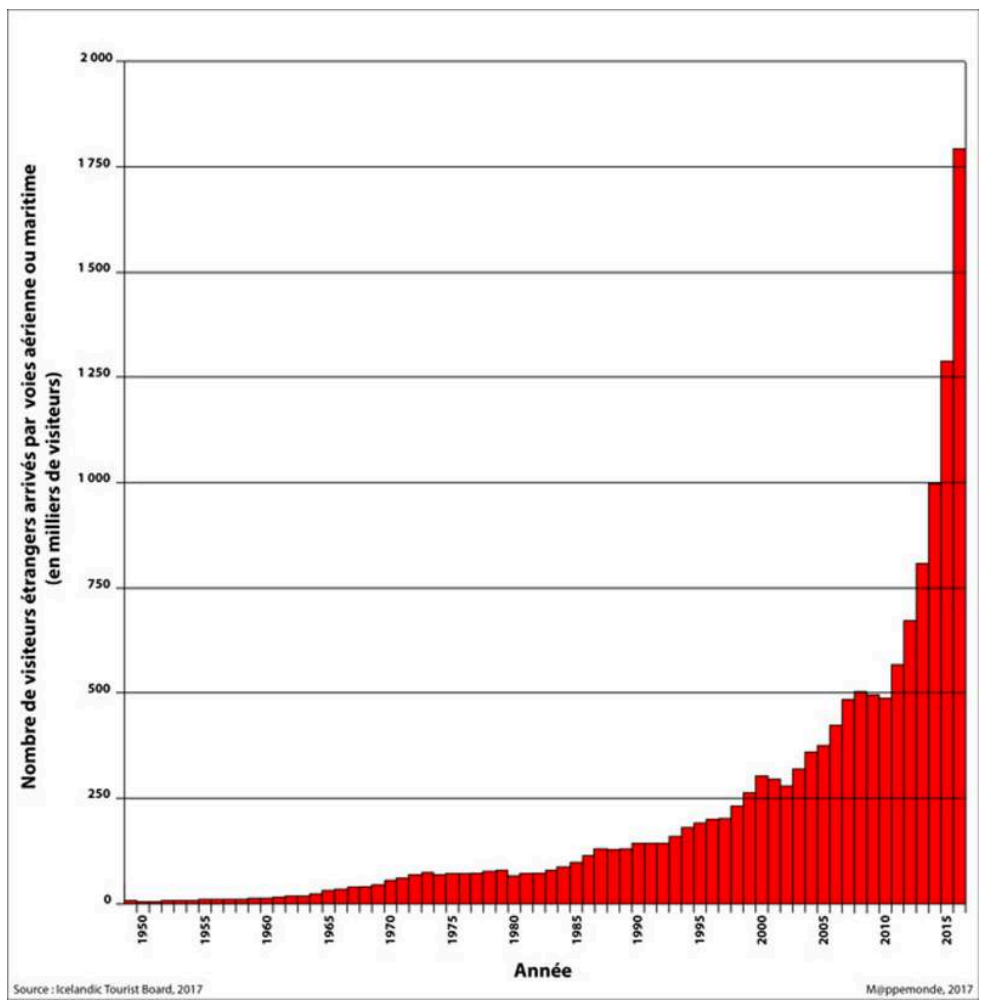

Source : Icelandic Tourist Board, 2017, https://www.ferdamalastofa.is/en/recearch-and-statistics/ numbers-of-foreign-visitors

Cet essor touristique participe grandement à une économie redevenue florissante $(+7 \%$ de croissance en 2016), alors que l'économie de l'île de glace avait brutalement plongé fin 2008 et début 2009 (-7\% lors de cette année, $-3,5 \%$ en 2010). Le taux de chômage a regagné son taux d'avant la crise (3\%) alors qu'il dépassait les $8 \%$ lors de ces années noires. Le niveau de vie avait chuté de 15,5\% en 2009, l'inflation s'était envolée en 2010 ( $17 \%$ contre $3 \%$ aujourd'hui) et l'effondrement de la couronne islandaise avait rendu le pays, au coût de la vie jusqu'alors très élevé, attractif (OCDE, 2017). Avec 25000 salariés, le tourisme a considérablement permis de se rapprocher du plein emploi et il constitue depuis 2013 la principale source de revenus de l'île.

La campagne de relance adoptée par le gouvernement en 2008, intitulée «Inspiré par l'Islande $»^{11}$ qui a pu surfer sur l'éruption de l'Eyjafjallajökull en 2010, largement médiatisée, puis sur celle du Grimsvötn en 2011, qui a toutefois moins défrayé la chronique, a contribué à faire connaître la destination Islande dans le monde (Huijbens et Benediktsson, 2013). Inspired by Iceland est devenu le slogan de l'agence de promotion du tourisme en Islande, Promote Iceland.

5 La situation de l'Islande comme pont entre deux continents a contribué à cet envol touristique : elle a constitué historiquement une étape sur les vols transatlantiques. Son intérêt géopolitique fut bien compris par les États-Uniens qui y ont installé une base de l'OTAN en 1951, fermée en 2006 ; l'actuel aéroport international de Keflavik est ainsi un ancien aéroport militaire. La durée des escales entre l'Europe et l'Amérique du Nord permet au voyageur en transit de s'arrêter quelques heures au Blue Lagoon (du nom d'un cocktail inventé en 1960 par le Harry's bar), fréquenté par les Chinois ( $6^{\mathrm{e}}$ nationalité parmi les visiteurs), les Indiens, les Japonais ou les Russes. Les États-Uniens, puis les 
Britanniques, suivis des Allemands, des Norvégiens et des Danois constituent les cinq premières nationalités représentées de touristes. D’ailleurs, la proximité du site de l'aéroport international fait qu'il est visible lors de tout atterrissage (photo 5). L'activité touristique reste toutefois largement saisonnière, l'aéroport international de Keflavik culminant à 291000 passagers en juillet 2016, et $40 \%$ des visiteurs se concentrant sur l'île durant les trois mois d'été (figure 2).

Figure 2. Carte de situation

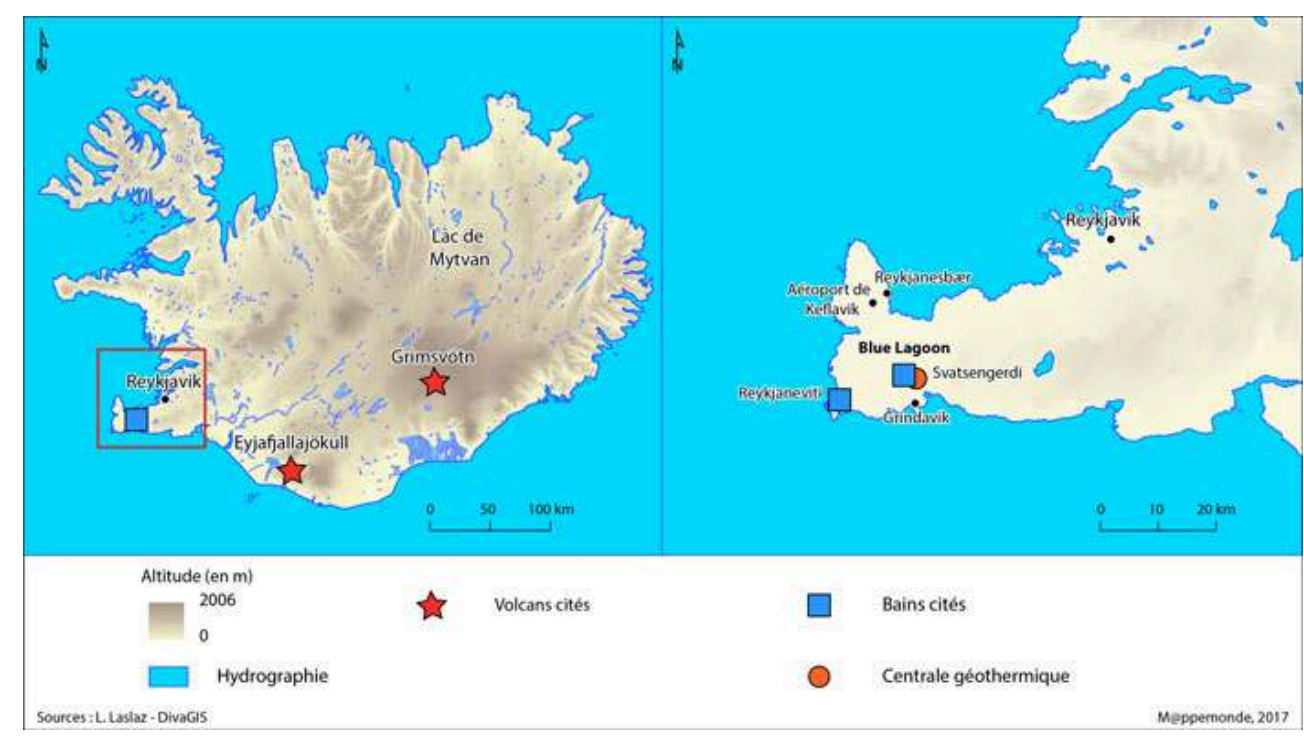

L. Laslaz, 2016

\section{Des touristes et de l'eau chaude}

6 Le bain, sur une île dont les côtes ne s'y prêtent guère, fait ainsi office de produit d'appel, notamment les bains chauds, comme cela est le cas ailleurs en Europe du Nord (Suède notamment) (photo 1). 
Photo 1. Couple s'immergeant dans une piscine d'eau chaude, Islande du Sud

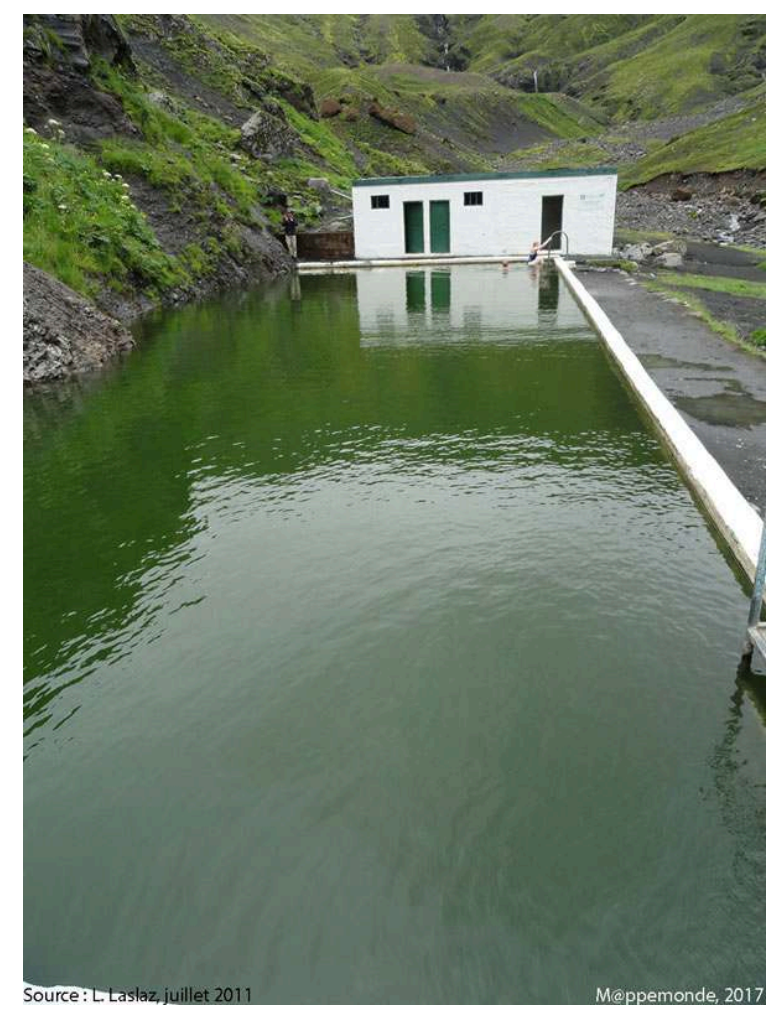

Le bâtiment correspond aux vestiaires.

L. Laslaz, juillet 2011

7 C'est ainsi le contraste entre le froid et le chaud qui est cultivé, que symbolisent le sauna (dans le Blue Lagoon, ce dernier occupe une forme de grotte taillée dans la lave) ou encore le bain chaud et l'air vivifiant qui est ainsi mis en scène (Vacher, 2012). Il constitue désormais un tel produit d'appel que la réservation en est recommandée, y compris dans la presse (figure 3). 
Figure 3. Publicité parue dans le journal promotionnel de la capitale

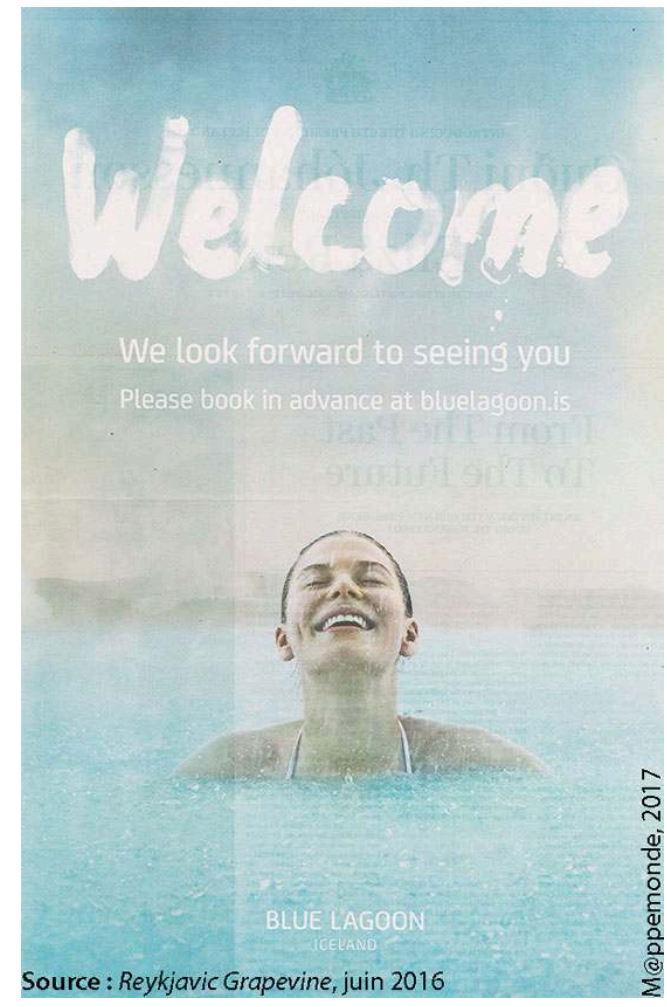

Reykjavik Grapevine, juin 2016

\section{Le Blue Lagoon ou l'improbable réussite d'une hétérotopie}

\section{Des opportunités du commerce de l'eau de mer...}

Indiscutablement, la réussite commerciale est au rendez-vous de ce complexe lancé en 1999. Mais le site n'avait pas à la base de finalités touristiques: il contient six millions de litres d'eau de mer résultant des forages de l'usine géothermique de Svatsengerdi, construite en 1976, qui pompent à deux kilomètres de profondeur (photo 2). Spontanément des baigneurs se rendaient dans l'étendue d'eau qui jouxtait l'usine et constataient les effets bénéfiques de cette immersion sur la peau, puis des bains ouvrirent officiellement en 1987. 
Photo 2. L'usine géothermique de Svatsengerdi (visible par son panache de fumée, municipalité de Grindavik) et le bassin d'eau chaude au premier plan

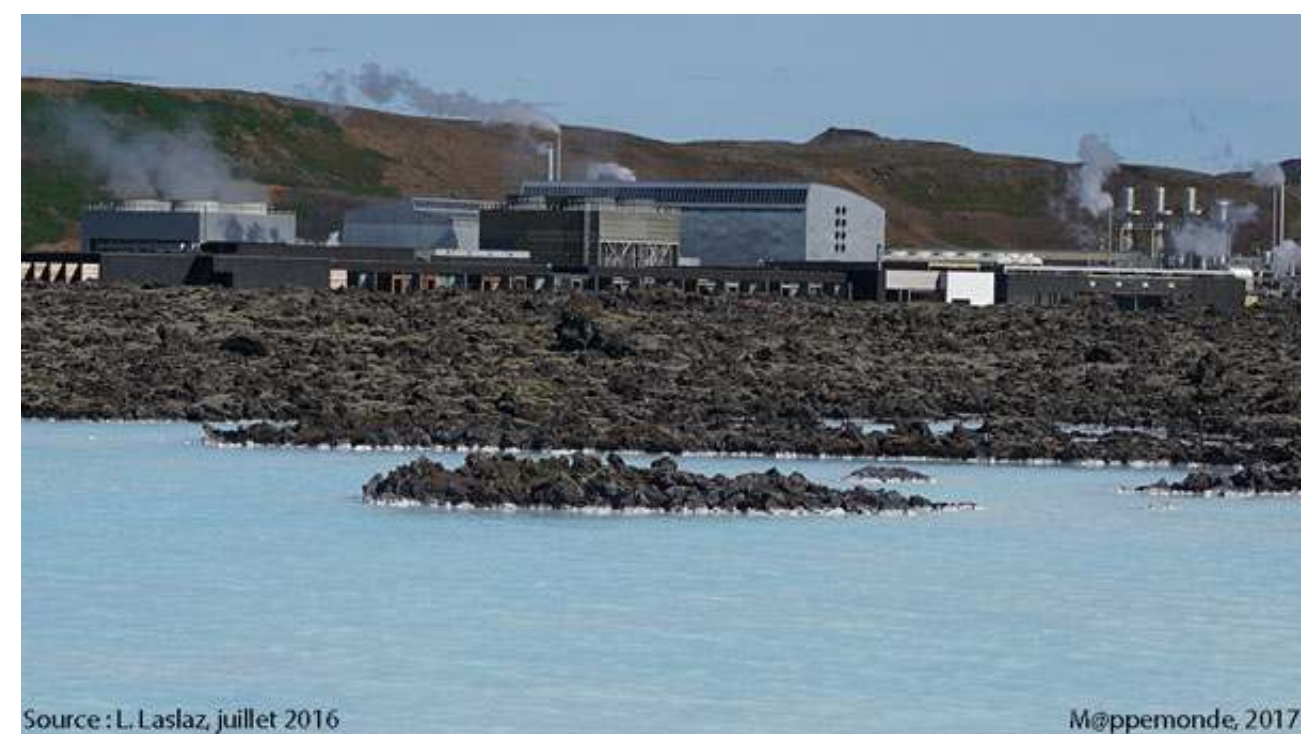

L. Laslaz, juillet 2016

9 L'eau arrive à $240^{\circ} \mathrm{C}$, génère de l'électricité et sert à chauffer de l'eau froide ; elle est relâchée dans un bassin de $5000 \mathrm{~m}^{2}$ qui comporte $75 \%$ d'eau de mer et $25 \%$ d'eau douce, renouvelée toutes les 40 heures. L'eau avoisine les 37 à $39^{\circ} \mathrm{C}$, ce qui explique que les bactéries n'y survivent pas et que l'ajout de chlore ou d'autres produits chimiques n'y soit pas utile. Ces eaux résiduelles sont riches en sels et minéraux, en silice ; cette dernière, combinée à la pureté de l'eau et à la présence d'algues bleues microscopiques, confère au bassin cette couleur bleu turquoise si caractéristique. Les effets sur la peau (lutte contre l'eczéma et le psoriasis) sont favorables, à tel point que le Blue Lagoon est reconnu comme lieu de traitement par les ministères de la santé islandais et danois. Quant au bâtiment, il s'agit d'un ensemble architectural inauguré en 1999 sur les plans de l'architecte Sigriður Sibórsdóttir, qui a nécessité 60000 à 70000 blocs de lave pour ériger ce complexe de $3000 \mathrm{~m}^{2}$ (photo 6). Face au succès, le lagon est déplacé en 1999, complété par un spa puis par une clinique en 2005.

\section{Des Blue Lagoon partout ? De l'eau pompée à l'eau mise en scène}

Blue Lagoon est devenu un copyright. Le Myvatn Nature Bath (photo 3) est situé au nord de l'île, a ouvert postérieurement (2004) à proximité du troisième plus grand lac de l'île ; il est bien moins cher ( $25 €$ en 2011, 35 en 2017), mais se base sur le même concept et constitue un duplicata beaucoup moins connu et accessible. Avec 8,6 \% des voyageurs à la journée, le lac Myvatn est la $16^{\mathrm{e}}$ destination de l'île, mais cela ne signifie pas que tous se rendent au complexe de baignade. Le bassin comporte 3,5 millions de litres d'eau à une température de 36 à $40^{\circ} \mathrm{C}$. 


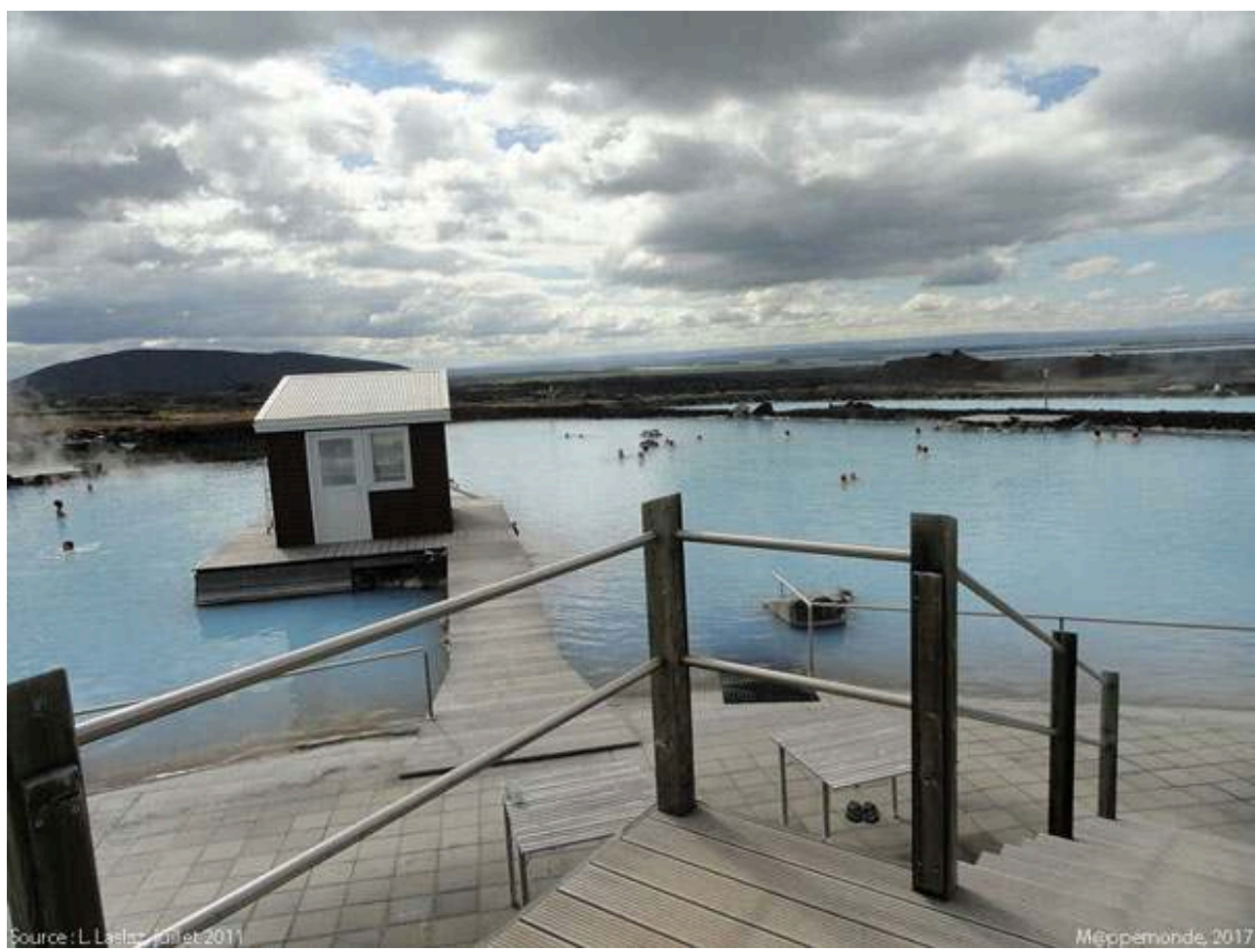

Accès au bassin situé dans l'un des sites géothermiques les plus actifs d'Islande.

L. Laslaz, juillet 2011

11 Minéraux, silice et micro-organismes sont censés soigner les clients à $100 \mathrm{~km}$ du cercle polaire arctique. Situé à $489 \mathrm{~km}$ et 6 heures 40 de route de la capitale, il est plus à l'écart des grands circuits touristiques et n'a pas constitué une marque commerciale comme le premier. Le Blue Lagoon est en effet distant de $49 \mathrm{~km}$ de la capitale et surtout de $20 \mathrm{~km}$ de l'aéroport, et accueille très peu d'Islandais, ce qui est aussi le cas du Myvatn Nature baths. Seulement $2 \%$ des Islandais se sont rendus au Blue Lagoon en 2015 contre 41,3 \% dans la deuxième ville du pays, Akureyri (Icelandic Tourist Board, 2016). Du côté des touristes, près de $8 \%$ s'y sont rendus en 2015 lors d'une excursion à la journée, contre 9,3 \% en 2012 (Icelandic Tourist Board, 2013), ce qui représente toutefois peu pour un séjour moyen de 14,7 nuits en 2015. Le Blue Lagoon était cependant le premier site cité par les touristes en hiver (35\%) en 2012.

Face à ce Blue Lagoon inachevé que constitue Myvatn Nature Bath, il existe également des Blue Lagoon "potentiels » dans le sens où la stratégie économique des exploitants géothermiques ne leur a pas encore donné cette forme. Ainsi, sur la presqu'île de Reykjanes, à $20 \mathrm{~km}$ au sud-ouest du Blue Lagoon, le site de Reykjaneviti (Reykjanes Power station ou Power Plant Earth, créée en 2006 près des sources chaudes de Gunnhuver) (photo 4) constitue un équivalent de Svatsengerdi d'un point de vue géothermique, avec rejet dans un bassin des eaux chaudes pompées en profondeur, mais celui-ci n'est pas à ce jour équipé pour des activités récréatives. 
Photo 4. Reykjanes Power station ou Power Plant Earth, sur la péninsule de Reykjanes, sud-ouest de l'Islande

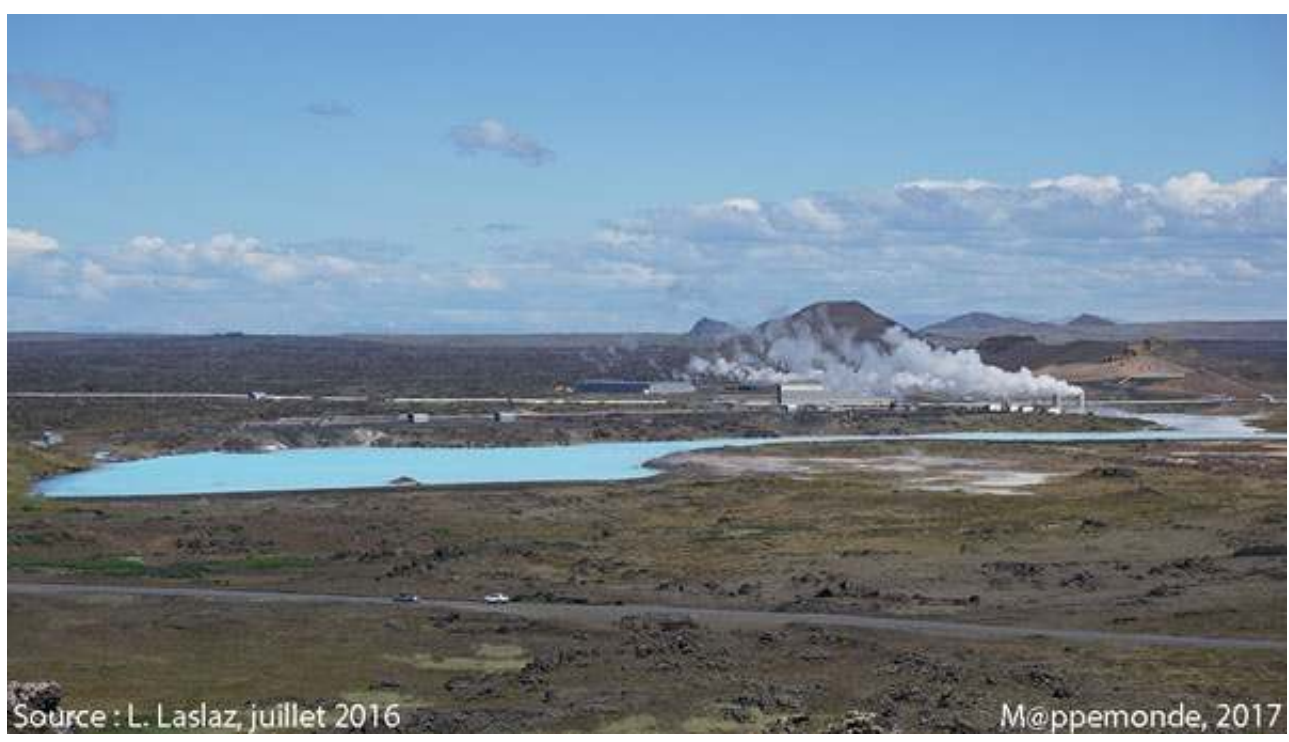

L. Laslaz, juillet 2016

13 Il constitue en quelque sorte l'envers du décor, le site de production énergétique avant sa complémentation récréative, car l'accessibilité et la situation expliquent le développement ou non de cette stratégie.

\section{Le Blue Lagoon comme « hétérotopie »}

14 Le Blue Lagoon apparaît comme une hétérotopie (Foucault, 1984), car c'est un lieu hors d'Islande : son extraterritorialité, sa configuration de type insulaire (une forme d'isolat au milieu d'une coulée de lave de 1226) tranchent avec les retombées économiques significatives qu'il génère, notamment pour la municipalité de Grindavik. Le site de baignade fait davantage office d'oasis que de lagon, mais il constitue la projection spatiale d'un double ailleurs, emboîté : le lagon, dans l'« île de glace et de feu», apparaît comme un site extra-insulaire : un lieu dans un autre lieu, en rupture. En ce sens, l'utopie qui caractérise les hétérotopies, doublée d'une hétérochronie traduisant un temps du hors quotidien, est tangible.

15 Sans doute la localisation d'une telle structure dans un espace artificialisé ou dans l'exubérance de Dubaï n'aurait-elle pas la même saveur aux yeux de ceux qui la fréquentent. Car c'est en partie par cette localisation et cette couleur singulières dans ce contexte paysager et septentrional qu'il détonne le plus. 


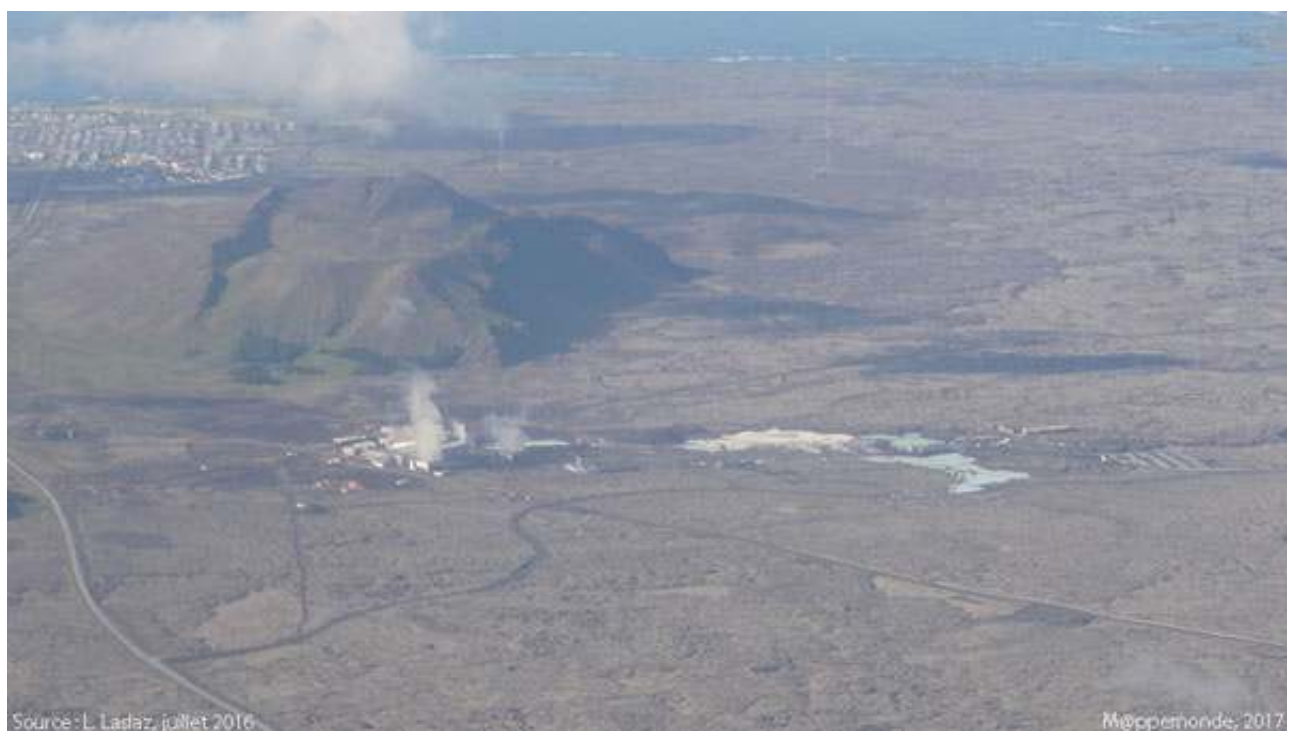

Le site géothermique, le complexe hôtelier, le bassin et son parking sont bien visibles à droite. En arrière-plan, Grindavik et l'Océan atlantique.

L. Laslaz, juillet 2016

\section{De l'inscription spatiale du complexe énergico- touristique à l'établissement d'une marque}

\section{Un lieu fermé}

16 Le complexe du Blue Lagoon apparaît comme un ensemble hermétique fermé par des murs de lave, puisque l'entrée est payante (photo 6). Michel Foucault (1984) insistait sur les systèmes d'ouverture et de fermeture des hétéropies, isolées des espaces environnants ; ici, la visée est clairement commerciale, mais elle participe aussi au sentiment de bien-être par le confinement et l'isolement, très relatif, car même si le nombre d'entrées payantes n'est pas rendu public par la société d'exploitation, la foule se presse, notamment durant les quatre mois d'été. 
Photo 6. Entrée du Blue Lagoon

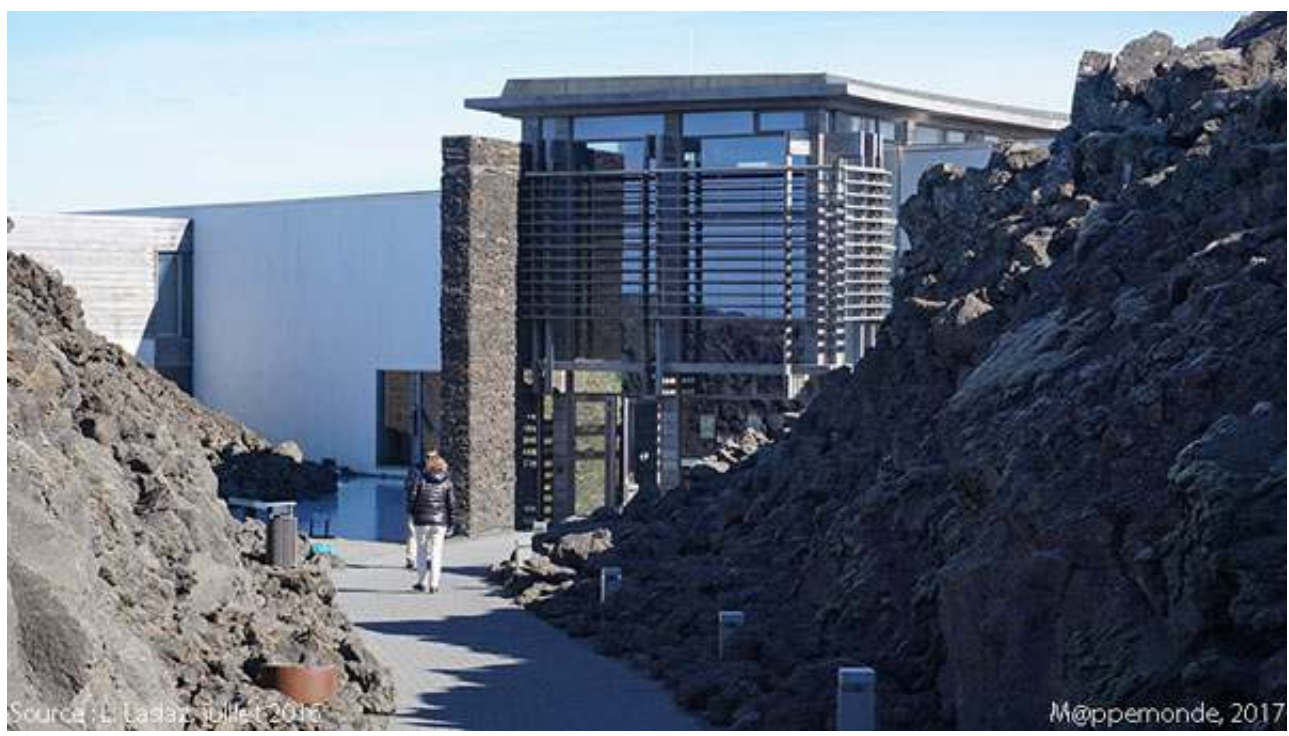

L. Laslaz, juillet 2016

17 Autour du spa et des bains se déploie tout un complexe touristique - bâti au fur et à mesure que la formule rencontrait un succès commercial - comportant une clinique, le restaurant Max et un hôtel (photo 7).

Photo 7. L'hôtel et le restaurant Max. En arrière-plan, l'usine géothermique

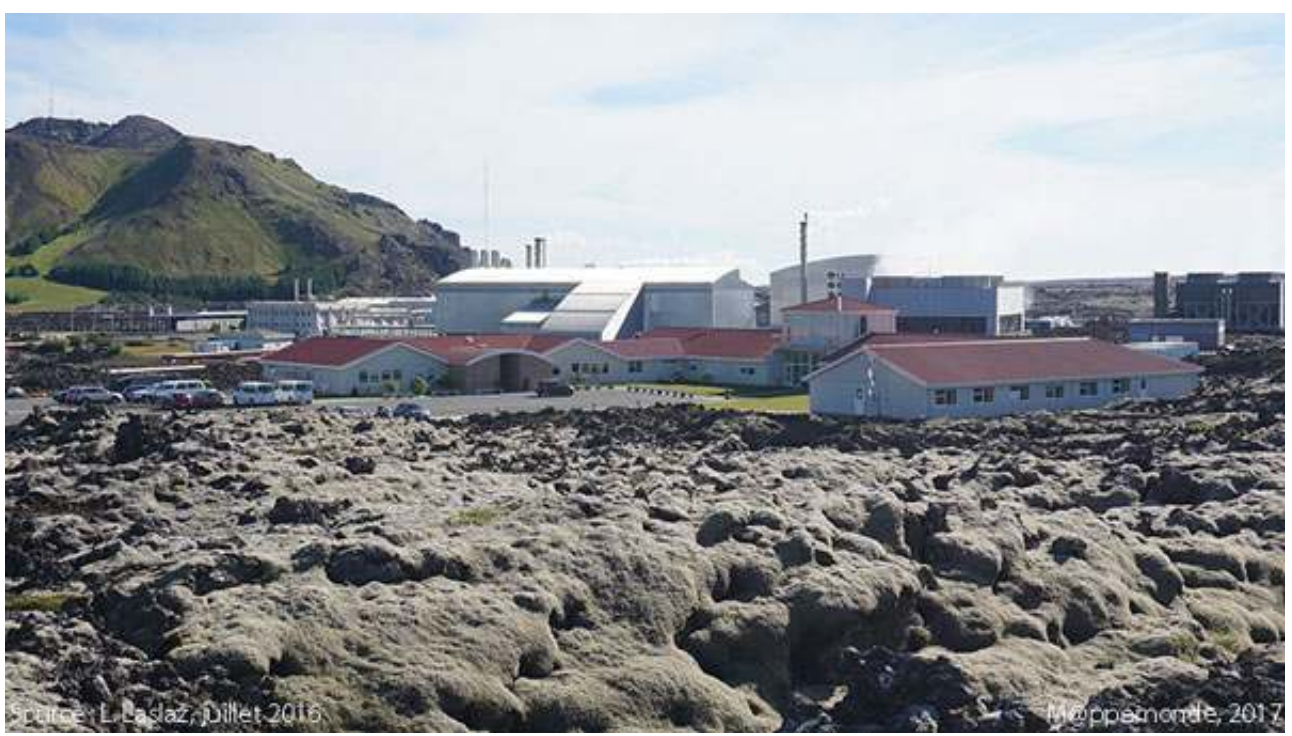

L. Laslaz, juillet 2016

\section{Une extension comme rançon du succès}

18 En janvier 2016, le lagon a été agrandi de $50 \%$ et occupe désormais $8700 \mathrm{~m}^{2}$ pour une capacité de neuf millions de litres (près de trois fois celle de Myvatn Nature Bath). L'ajout de nouveaux services (espace de tranquillité et de relaxation, traitements par les eaux du spa) s'est fait en lien avec la construction d'un nouvel ensemble comprenant un hôtel de luxe de 60 chambres comportant des salles de conférences. Cette construction 
s'est opérée sous la houlette de la même architecte depuis le lancement du Blue Lagoon, Sigriður Sibórsdóttir, dont le travail est présenté comme associant les formes naturelles et les productions humaines. L'affichage d'un cadre « naturel », sur lequel on peut s'interroger au regard du caractère totalement anthropique du dispositif, est ainsi une constante du discours commercial mis en œuvre par le complexe.

Photo 8. Les travaux d'extension du parking

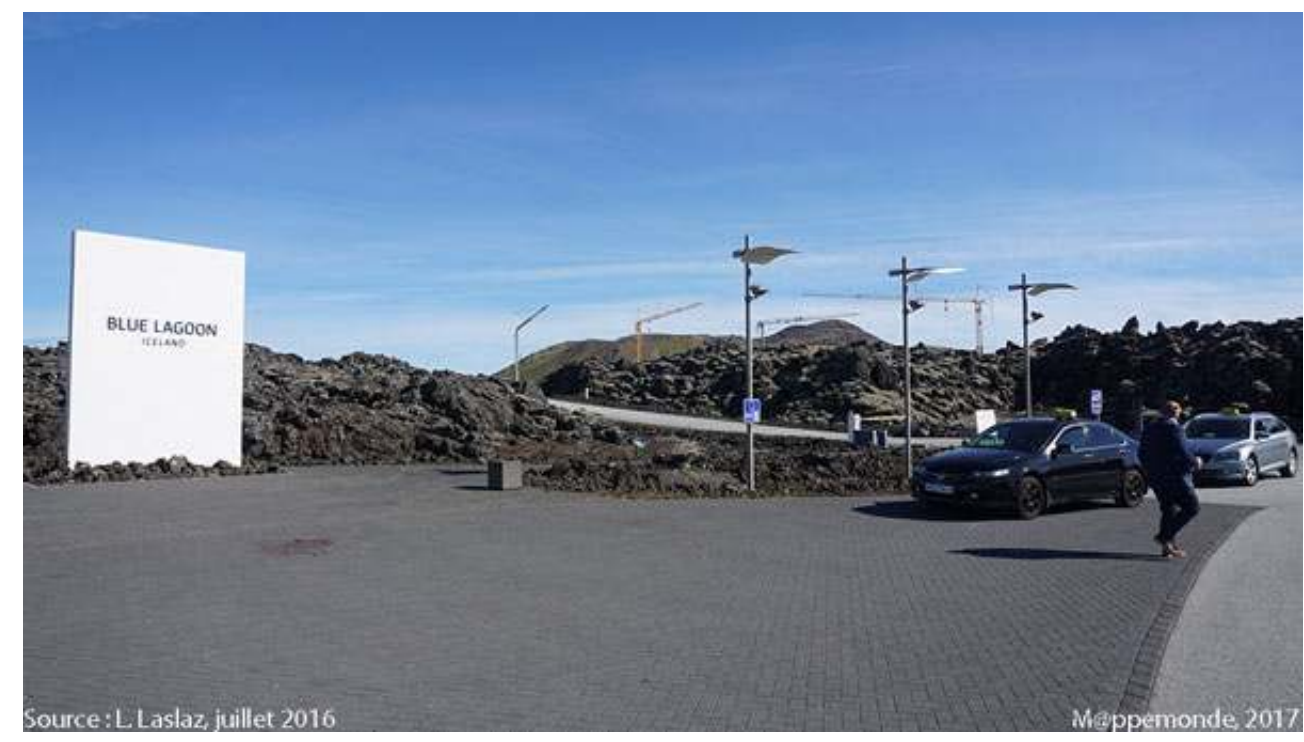

La construction d'un spa et d'un hôtel de luxe ouverts en 2017 se devinent à travers les quatre grues en arrière-plan

L. Laslaz, juillet 2016

Photo 9. Les désagréments liés aux travaux font l'objet d'explications et d'excuses des gestionnaires dans tout le complexe, jusqu'au bord du bassin

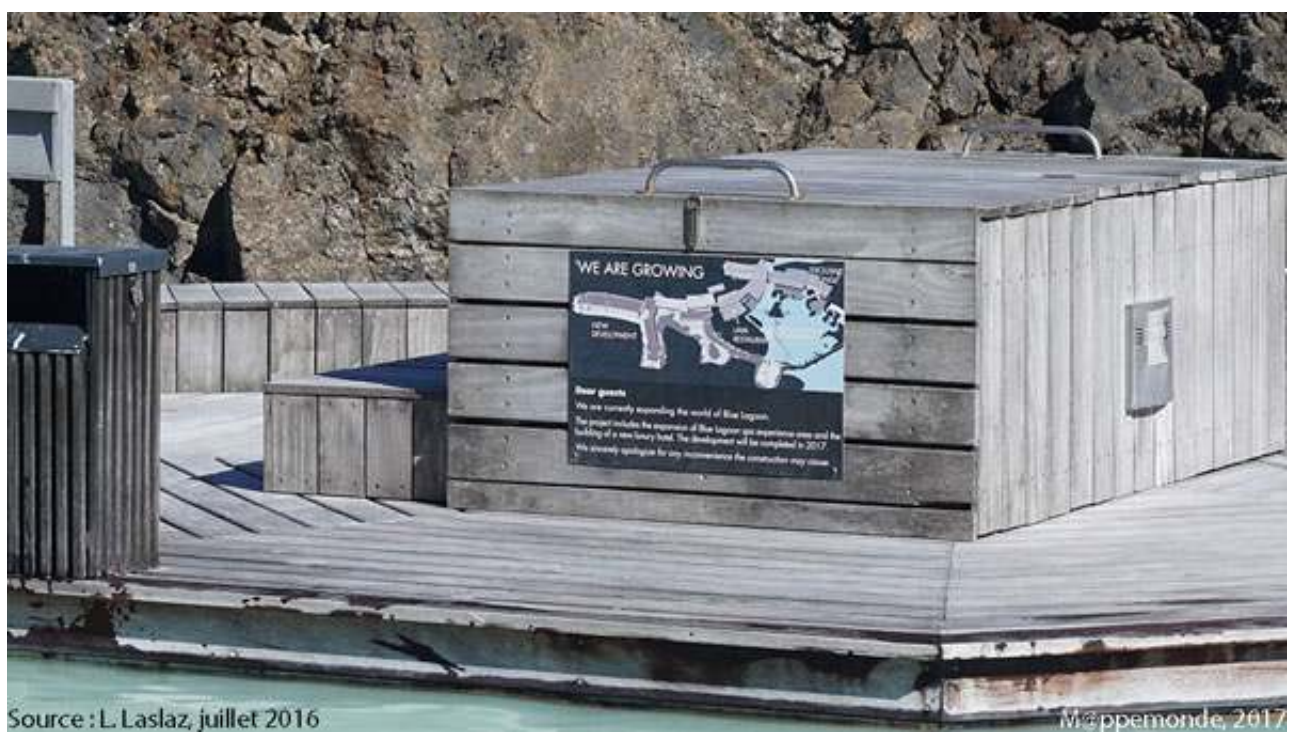

L. Laslaz, juillet 2016

Une autre évolution significative du soin apporté à la mise en scène du lieu et au confort des clients entre 2011 et 2016 repose sur l'installation de bars enterrés dans le 
bassin, pour que le comptoir soit à hauteur du baigneur et qu'il puisse s'adresser directement aux serveurs qui l'attendent et commander auprès d'eux.

Photo 10. Les clients au niveau du comptoir du bar

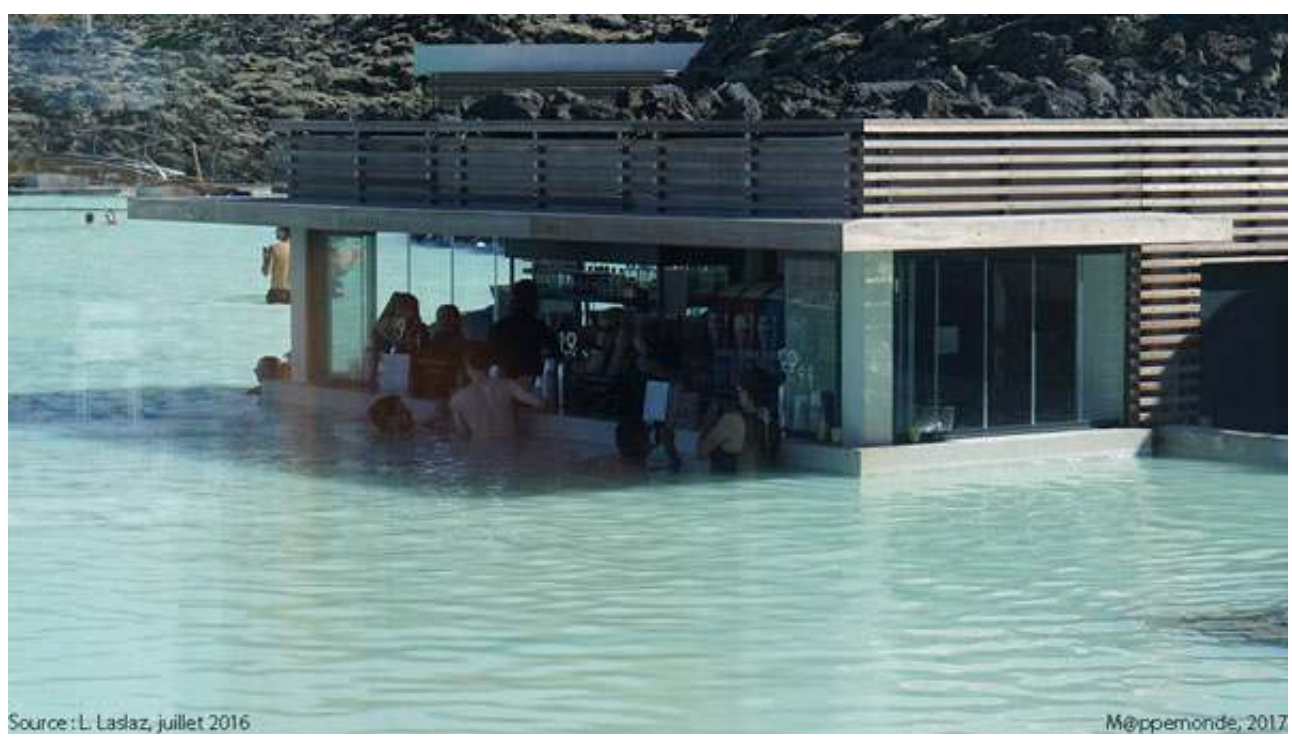

L. Laslaz, juillet 2016

Photo 11. Le bar au fond du lagon ; les élévations de lave et l'usine géothermique en arrière-plan

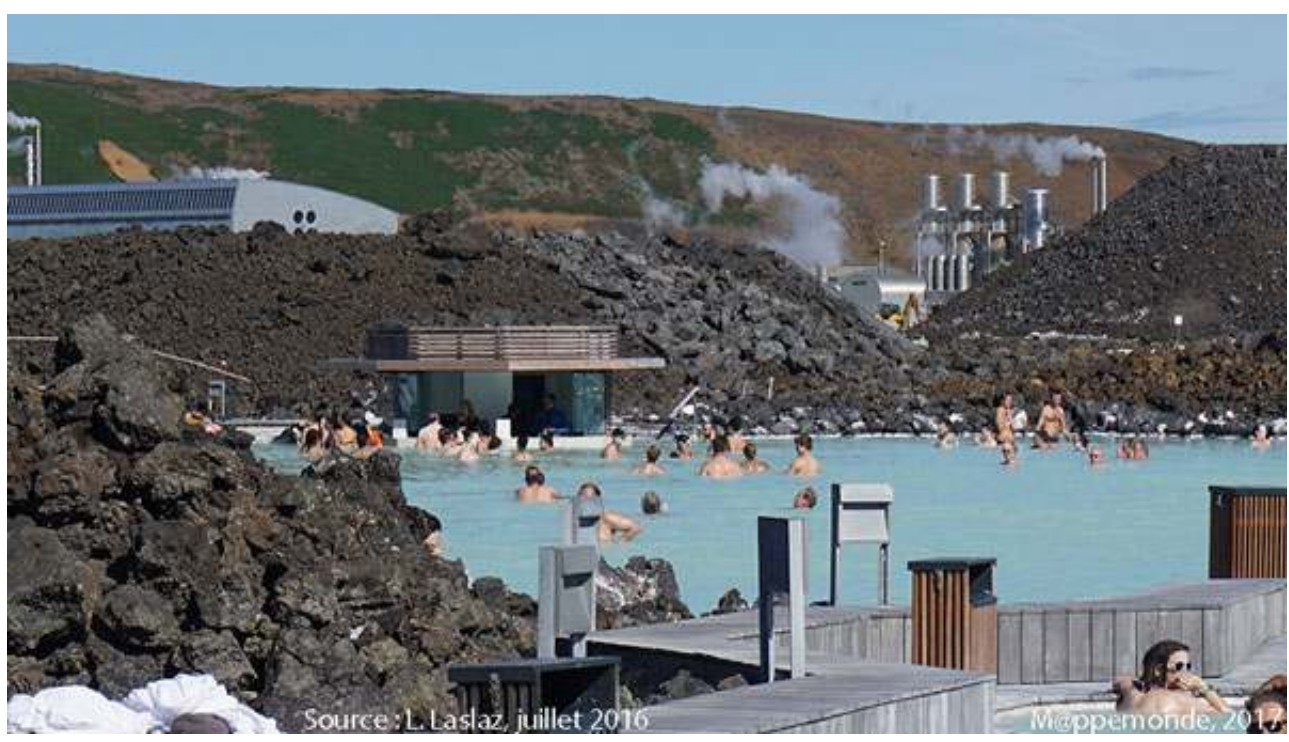

L. Laslaz, juillet 2016

\section{La piscine chauffée la plus chère du Monde?}

Le prix d'entrée du Blue Lagoon est très dissuasif : $60 €$ comme tarif de base (serviette et «masque de boue », mais que l'on ramasse librement au fond des bassins) ; $75 €$ comme $2^{\mathrm{e}}$ formule et $90 €$ comme $3^{\mathrm{e}}$ formule, ce qui en fait une des piscines chauffées les plus chères du monde. Les prix, pour éviter tout effet dissuasif, ne sont d'ailleurs plus 
affichés en 2016 par rapport à 2011 où ils étaient davantage accessibles (contrecoup de la crise et rançon du succès obligent).

\section{L'établissement d'une marque de produits de soin}

21 Chez Michel Foucault (1984), l'hétérotopie est aussi le lieu qui efface et purifie les autres. En ce sens, la détente permise par le bassin, les soins apportés en son sein confortent ce sentiment d'hétérotopie, cultivée par ses gestionnaires. Car au-delà du complexe de spa et de la clinique, le Blue Lagoon est devenu une marque qui ne repose pas que sur la pratique de la baignade reconnue pour ses vertus curatives. Elle s'étoffe par le biais de produits dérivés ou de services annexes : prestations de soins, produits de beauté (lignes de sels marins, brumisateurs et lotions fabriqués à partir de boues de silice, d'algues et minéraux extraits du lagon sont à la vente dans le magasin du Blue Lagoon, photo 12), restaurant, auxquels s'ajoutent une salle de conférence et un centre de recherche et de développement qui travaille sur les produits de soin qui sont commercialisés. Cette marque sert tout autant de produit d'appel pour l'Islande, comme à l'aéroport où la publicité du complexe est présente partout (photo 13). Quant aux produits qui en émanent, ils disposent aussi d'une boutique dédiée au sein de la zone duty free (photo 14).

Photo 12. Entrée du Blue Lagoon et magasin sur la gauche

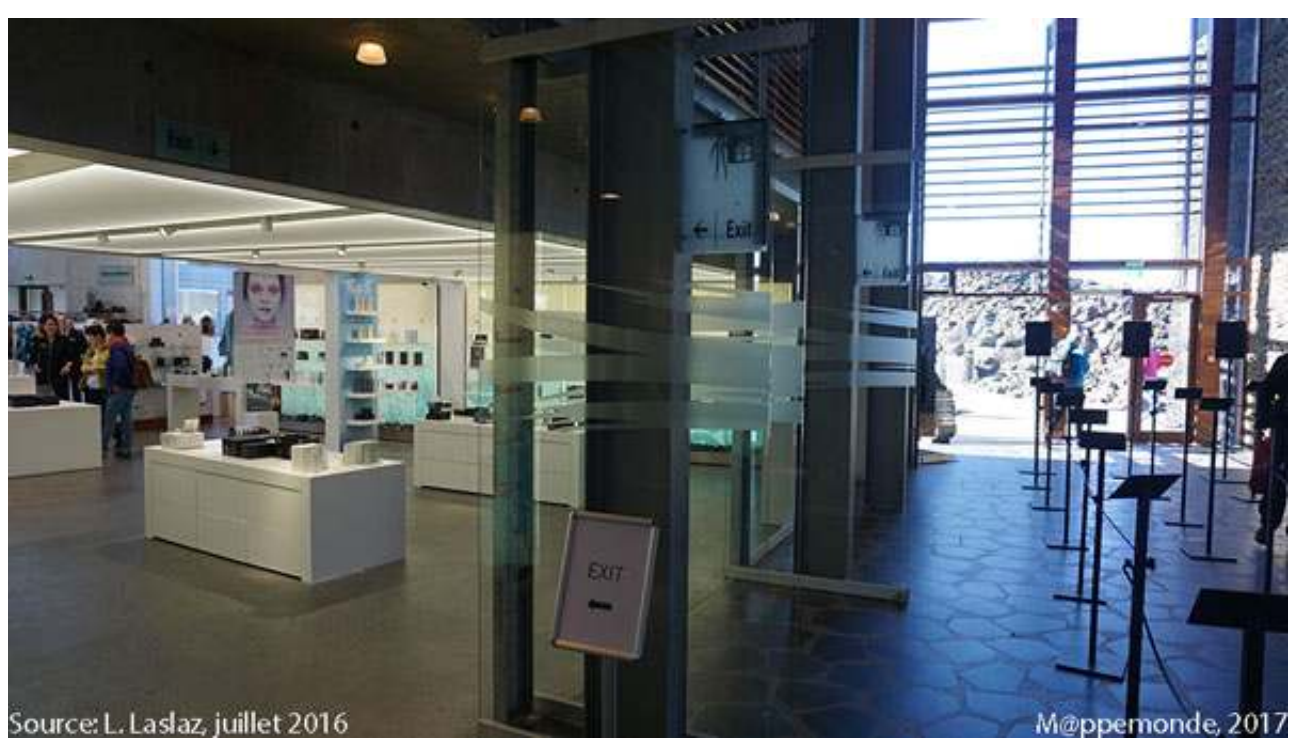

L. Laslaz, juillet 2016 
Photo 13. Couloir de l'aéroport international de Keflavik, Islande

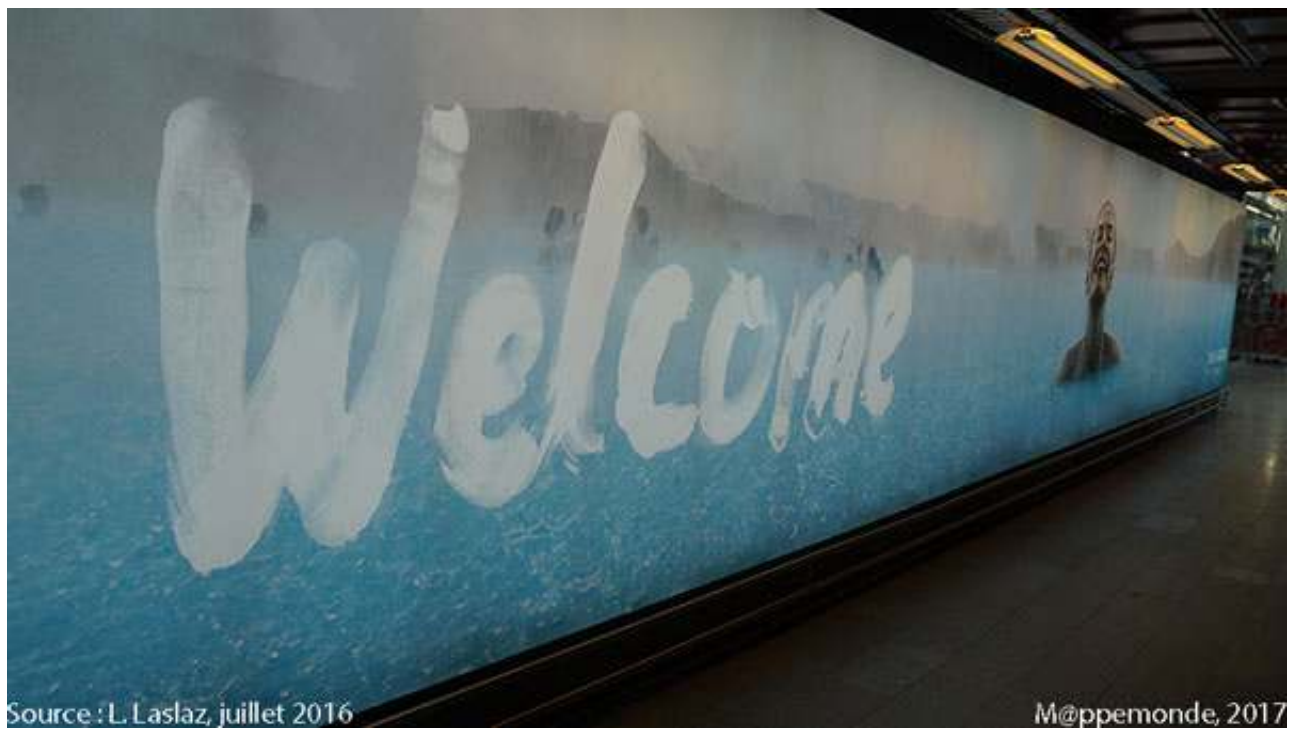

L. Laslaz, juillet 2016

Photo 14. Magasin dans la zone duty free de l'aéroport international de Keflavik, Islande

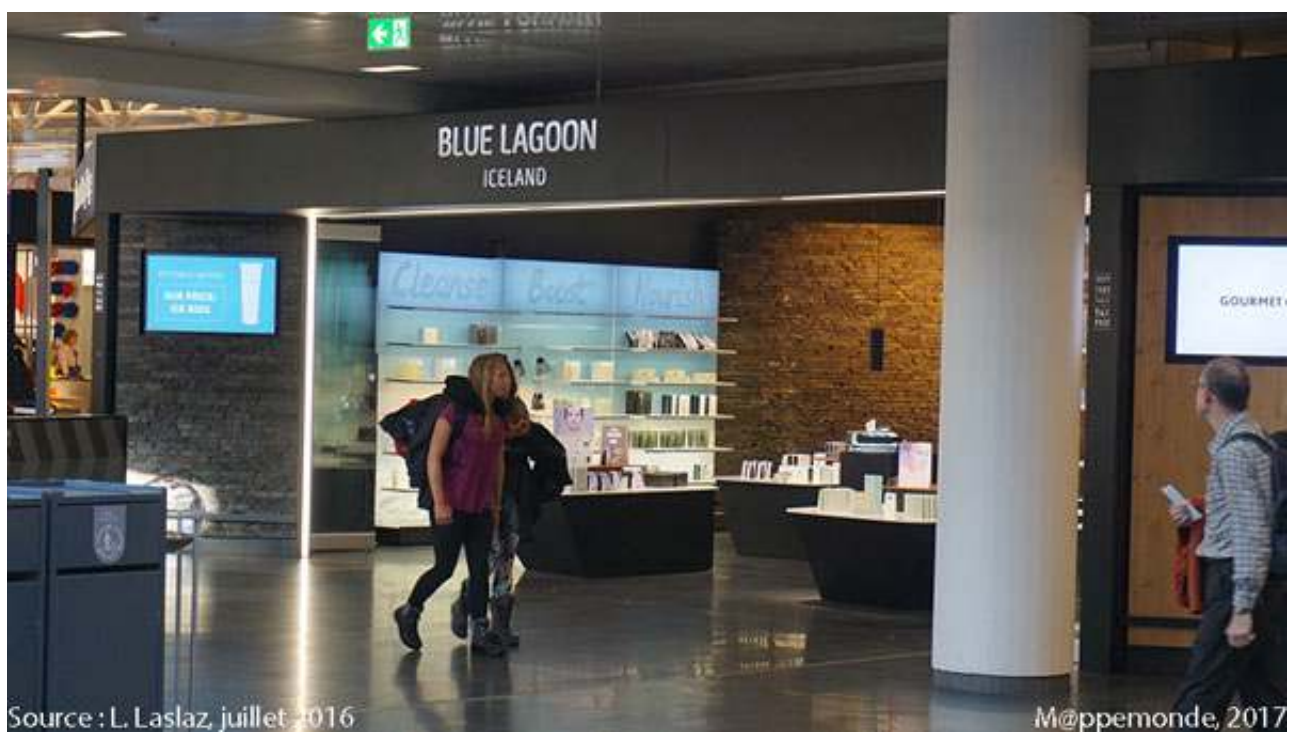

L. Laslaz, juillet 2016

\section{Conclusion}

22 Si le site du Blue Lagoon est loin de l'image de nature qui revient dans $75 \%$ des réponses des touristes comme raison de leur visite en Islande (Icelandic Tourist Board, 2016), il procure néanmoins le sentiment de bien-être et de contraste lié à un cadre singulier recherché par ces voyageurs et largement mis en avant dans la promotion touristique de l'île. Si l'Islande fait office d'espace autre pour la plupart des touristes qui s'y rendent, alors le Blue Lagoon constitue cette forme de superlatif de l'hétérotopie. 
air des acteurs commerciaux de l'île aura été de faire d'un site géothermique, sans grand intérêt récréatif, une des premières destinations touristiques de l'île (les Islandais préférant toutefois les «bains de nature » gratuits) et, bien au-delà, un identifiant très fort et un incontournable de tout séjour sur l'île de glace.

\section{BIBLIOGRAPHIE}

FOUCAUlT M. (1984). « Des espaces autres ». Conférence au Cercle d'études architecturales, 14 mars 1967. Architecture, Mouvement, Continuité, n 5, octobre 1984, p. 46-49 (publié in FOUCAUlT M., 2001, Dits et Écrits, II, 1976-1988, Gallimard, 1736 p.).

HUIJBENS E.H., BENEDIKTSSON K. (2013). “Inspiring the visitor? Landscapes and horizons of hospitality". Tourist Studies, vol. 13, n² 2, p. 189-208.

Icelandic Tourist Board, mai 2013, Tourism in Iceland in figures, 26 p.

Icelandic Tourist Board, mai 2016, Tourism in Iceland in figures, 26 p.

JÓHANNESSON G.T., HUIJBENS E.H. (2010). “Tourism in times of crisis: exploring the discourse of tourism development in Iceland". Current Issues in Tourism, vol. 13, n 5, p. 419-434.

LASLAZ L. (2016). « Kárahnjúkar, le diable dans l'éden. Hydroélectricité et espaces protégés en Islande ». Géoconfluences, image à la une, septembre 2016. En ligne : http://geoconfluences.enslyon.fr/informations-scientifiques/a-la-une/image-a-la-une/image-a-la-une-karahnjukar-islande OCDE (2017). Economic surveys of Iceland. Preserving sustainable and inclusive growth. En ligne : http://www.oecd.org/eco/surveys/Iceland-2017-OECD-economic-survey-preserving-sustainableand-inclusive-growth.pdf

VACHER L. (2012). « La découverte récréative des eaux tropicales... et on inventa l'eau chaude et le blue lagoon ». Les Cahiers d'outre-mer, $n^{\circ} 260$, p. 555-567. https://journals.openedition.org/com/ 6749

\section{Sitographie}

Myvatn Nature baths : https://myvatnnaturebaths.is/

Blue Lagoon : http://www.bluelagoon.com/, annoncé en toute modestie comme « une des 5 merveilles du monde».

Péninsule de Reykjanes : https://www.visitreykjanes.is/

\section{NOTES}

1. http://www.inspiredbyiceland.com/ 


\section{AUTEUR}

\section{LIONEL LASLAZ}

Maître de conférences HDR en géographie UMR 5204 EDYTEM (Environnements, Dynamiques et Territoires de la Montagne) CNRS/Université Savoie Mont-Blanc 